\title{
Measurable residual disease status and outcome of transplant in acute myeloid leukemia in second complete remission: a study by the acute leukemia working party of the EBMT
}

\author{
Maria H. Gilleece (1) ${ }^{1}$, Avichai Shimoni $\mathbb{0}^{2}$, Myriam Labopin ${ }^{3}$, Stephen Robinson ${ }^{4}$, Dietrich Beelen ${ }^{5}$, Gerard Socié6 \\ Ali Unal ${ }^{7}$, Arnold Ganser ${ }^{8}$, Antonin Vitek ${ }^{9}$, Henrik Sengeloev ${ }^{10}$, Ibrahim Yakoub-Agha $\mathbb{B}^{11}$, Eleni Tholouli ${ }^{12}$, \\ Emmanuelle Polge ${ }^{13}$, Mohamad Mohty $\mathbb{B D}^{14}$ and Arnon Nagler ${ }^{15}$
}

\begin{abstract}
Measurable residual disease (MRD) prior to hematopoietic cell transplant (HCT) for acute myeloid leukemia (AML) in first complete morphological remission (CR1) is an independent predictor of outcome, but few studies address CR2. This analysis by the Acute Leukemia Working Party of the European Society for Blood and Marrow Transplantation registry assessed HCT outcomes by declared MRD status in a cohort of 1042 adult patients with AML CR2 at HCT. Patients were transplanted 2006-2016 from human leukocyte antigen (HLA) matched siblings $(n=719)$ or HLA 10/10 matched unrelated donors $(n=293)$. Conditioning was myeloablative $(n=610)$ or reduced-intensity $(n=432)$ and 566 patients (54\%) had in-vivo T cell depletion. At HCT, 749 patients (72\%) were MRD negative (MRD NEG) and 293 (28\%) were MRD positive (MRD POS). Time from diagnosis to HCT was longer in MRD NEG than MRD POS patients (18 vs. 16 months $(P<0.001)$. Two-year relapse rates were $24 \%(95 \% \mathrm{Cl}, 21-28)$ and $40 \%(95 \% \mathrm{Cl}, 34-46)$ in MRD NEG and MRD POS groups $(P<0.001)$, respectively. Leukemia-free survival (LFS) was 57\% (53-61) and $46 \%(40-52 \%)$, respectively $(P=0.001)$, but there was no difference in terms of overall survival. Prognostic factors for relapse and LFS were MRD NEG status, good risk cytogenetics, and longer time from diagnosis to HCT. In-vivo T cell depletion predicted relapse.
\end{abstract}

\section{Introduction}

Relapse of acute myeloid leukemia (AML) following allogeneic hemopoietic cell transplant (HCT) remains a major cause of treatment failure and indicates the presence of persistent subclinical disease, despite morphological complete remission (CR) at the time of transplant ${ }^{1-4}$. AML is a heterogeneous malignancy associated with a wide variety of fusion genes, mutations, and

Correspondence: Maria H. Gilleece (mgilleece@nhs.net)

${ }^{1}$ Leeds Teaching Hospitals Trust, St James's University Hospital, Leeds LS9 7TF, United Kingdom

${ }^{2}$ Hematology and Bone Marrow Transplantation Division, Chaim Sheba Medical Center, Tel-Hashomer, Sackler School of Medicine, Tel-Aviv University, 6997801 Tel-Aviv, Israel

Full list of author information is available at the end of the article

These authors contributed equally: Maria H. Gilleece, Avichai Shimoni overexpressed genes ${ }^{5-7}$. Multiple techniques such as multi-parameter flow cytometry immunophenotyping, real-time quantitative polymerase chain reactions, or high throughput sequencing are available to detect so-called "measurable residual disease" (MRD) in the presence of morphological $\mathrm{CR}^{8,9}$. Multi-parameter flow cytometry immunophenotyping MRD assays are applicable to $90 \%$ of patients with AML and may detect cells with a leukemiaassociated immunophenotype or a "different from normal" immunophenotype at a sensitivity of $10^{-3}$ to $10^{-5}$ in bone marrow ${ }^{5,10-15}$. In addition, up to $60 \%$ of young adults have a molecular marker detectable by real-time quantitative polymerase chain reactions assays and most cases of AML are amenable to molecular tracking by high throughput sequencing assays with sensitivity $10^{-4}$ to

\section{(c) The Author(s) 2021}

(c) (i) Open Access This article is licensed under a Creative Commons Attribution 4.0 International License, which permits use, sharing, adaptation, distribution and reproduction cc) in any medium or format, as long as you give appropriate credit to the original author(s) and the source, provide a link to the Creative Commons license, and indicate if changes were made. The images or other third party material in this article are included in the article's Creative Commons license, unless indicated otherwise in a credit line to the material. If material is not included in the article's Creative Commons license and your intended use is not permitted by statutory regulation or exceeds the permitted use, you will need to obtain permission directly from the copyright holder. To view a copy of this license, visit http://creativecommons.org/licenses/by/4.0/. 
$10^{-63}$. Thus MRD assessments may be used to assess the kinetics of response to therapy, as well as impending relapse, and have become an integral part of present-day clinical trials in $\mathrm{AML}^{3,16}$.

MRD status after induction therapy is prognostic of outcome in AML independent of other accepted risk parameters ${ }^{6,10,12,14,16-26}$. Furthermore, multiple large studies have demonstrated the prognostic importance of MRD pre-HCT in CR1 terms of subsequent relapse incidence (RI), leukemia-free survival (LFS), and overall survival $(\mathrm{OS})^{6,14,27-35}$. A previous study undertaken by the European Society for Blood and Marrow Transplantation (EBMT) found that patients with MRD at HCT had inferior survival at 2 years after HCT compared to those with an MRD negative status $(56.2 \%$ vs. $70 \%$ in adults aged less than 50 years and $50.7 \%$ vs. $62.1 \%$ in patients 50 years and older $)^{35}$.

HCT is usually deferred for patients with AML in CR1 if the relapse risk is less than $35 \%$ although only a minority of relapsing patients will achieve CR2 and proceed to $\mathrm{HCT}^{4,36-39}$. Breems et al. identified that the duration of CR1, age at relapse, cytogenetic risk factor at diagnosis, and a prior allogeneic HCT could be used to predict the likelihood of $\mathrm{CR} 2{ }^{40}$. These observations are consistent with other large studies ${ }^{41-45}$.

Those patients who proceed to HCT in CR2 have similar outcomes to patients transplanted in CR1 with a reported survival of $58.2 \%$ at 2 years after $\mathrm{HCT}^{45}$. Walter et al showed as part of a sub-set analysis that in 70 patients with AML in CR2 treated with myeloablative (MAC) HCT, 3 year OS post-HCT was $73 \%$ in MRD negative (MRD NEG) vs. 44\% in MRD positive patients $(\mathrm{MRD} P O S)^{31}$. In the first large series to address the impact of MRD status in AML CR2, we have analyzed the results of allogeneic HCT utilizing a large cohort of patients for whom MRD data had been deposited in the registry of the EBMT.

\section{Methods}

\section{Study design and data collection}

The Acute Leukemia Working Party of the EBMT approved and conducted this study. The EBMT supports data registration from more than 600 transplant centers, predominantly located within Europe. Centers are required to report all $\mathrm{HCT}$ with subsequent annual follow-up. EBMT Med A/B standardized data collection forms are completed and submitted to the registry by transplant center personnel following written informed consent from patients in accordance with Center ethical research guidelines ${ }^{46}$. Accuracy of data is assured by the individual transplant centers and by quality control measures such as regular internal and external audits. Since January 1, 2003, all transplant centers have been required to obtain written informed consent prior to data registration with the EBMT, following the Helsinki Declaration of 1975.

The objectives of the study were to assess the impact of MRD status on transplant outcomes in patients with AML CR2 at the time of transplant.

\section{Eligibility criteria}

Eligibility criteria were age $\geq 18$ years, first allogeneic HCT 2006-2016, a diagnosis of de novo AML in CR2 (excluding acute promyelocytic leukemia), and availability of MRD status prior to HCT as declared by the center. A recent survey of EMBT centers indicated that most used a combination of validated MRD assays as directed by the presence of specific mutations detectable by PCR and/or leukemia profiles amenable to detection by flow cytometry ${ }^{47}$. Cytogenetic status was classified using MRC UK criteria while any identified molecular markers at diagnosis were also noted ${ }^{48}$. Donors were restricted to a human leukocyte antigen (HLA) matched sibling donor (MSD) or volunteer unrelated donor with HLA match 10/ 10 (MUD). The graft source included peripheral blood stem cells (PBSC) or bone marrow grafts. Engraftment was assessed by conventional EBMT standards ${ }^{46}$. The intensity of conditioning and chronic GVHD were classified in accordance with published criteria ${ }^{49-52}$.

Patient, disease, and transplant-related characteristics for the two cohorts (MRD POS/ MRD NEG) were compared by using $X^{2}$ statistics for categorical variables and the Mann-Whitney test for continuous variables. The primary endpoint was leukemia-free survival (LFS). Secondary endpoints were relapse incidence (RI), non-relapse mortality (NRM), OS, acute graft-vs.-host disease (aGVHD), and chronic graft-vs.-host-disease (cGVHD), GVHD-free/relapse-free survival (GRFS). LFS was defined as survival with no evidence of relapse or progression. Relapse was defined as the presence of $5 \%$ bone marrow blasts and/or reappearance of the underlying disease. NRM was defined as death without evidence of relapse or progression. OS was defined as the time from alloSCT to death, regardless of the cause. GRFS was defined as events including grade 3-4 acute GVHD, extensive chronic GVHD, relapse, or death in the first post-HCT year ${ }^{53}$. Cumulative incidence was used to estimate the endpoints of NRM, RI, acute and chronic to accommodate for competing risks. To study acute and chronic GVHD, we considered relapse and death to be competing events. Probabilities of OS, LFS, and GRFS were calculated using the Kaplan-Meier method. Univariate analyses were done using Gray's test for cumulative incidence functions and the log-rank test for OS, GRFS, and LFS. A Cox proportional hazards model was used for multivariate regression. All variables differing significantly between the 2 groups or factors associated with one outcome in univariate analysis were included in the Cox model. In order to test 
for a center effect, we introduced a random effect or frailty for each center into the model ${ }^{54,55}$. Results were expressed as the hazard ratio (HR) with a $95 \%$ confidence interval $(95 \% \mathrm{CI})$. All tests were 2 -sided. The type I error rate was fixed at 0.05 for the determination of factors associated with time-to-event outcomes. Subgroup analyses were stratified by donor type (MSD or MUD). Statistical analyses were performed with SPSS 24.0 (SPSS Inc, Chicago, IL, USA) and R 3.4.0 (R Core Team (2017). R: A language and environment for statistical computing. $\mathrm{R}$ Foundation for Statistical Computing, Vienna, Austria. URL https://www.R-project.org/.)

\section{Results}

\section{Patient, donor, and transplant characteristics}

A total of 1042 patients satisfied the entry inclusion criteria for the study and of these, 293 had evidence of MRD at transplant. Patient and donor characteristics are summarized in Table 1.

When considered as two groups, MRD negative vs. MRD positive, the median age at transplant was 49 years in each. Karnofsky Performance Status and distribution of cytogenetic risk categories were also equivalent. There was a preponderance of male patients and donors overall. CMV serological status of patients was similar and although more CMV sero-negative donors were selected for MRD positive than MRD negative patients, this did not reach statistical significance. HLA-matched siblings were more likely to be selected as donors for MRD negative than MRD positive patients but this was not statistically significant. Both groups of patients were more likely to receive MAC HCT than RIC HCT. Time from diagnosis to transplant was shorter for MRD positive (median 15.9 months) than MRD negative recipients (18.2 months, $P<10^{-4}$ ) but median follow-up after transplant was similar at just over two years.

Conditioning regimens are listed in Supplementary Table 1. The commonest MAC HCT regimens were based on busulfan or TBI while RIC HCT regimens favored fludarabine, particularly when paired with busulfan or melphalan. GVHD prophylaxis is summarized in Supplementary Table 2 and included in vivo $\mathrm{T}$ cell depletion in a majority of transplants with equivalent usage in MRD negative (53\%) and MRD positive $(60 \%)$ recipients $(P=0.066)$. Otherwise, GVHD prophylaxis was based on calcineurin inhibitors with a majority of patients being treated with ciclosporin-based regimens.

\section{Transplant outcomes}

Transplant outcomes for the entire cohort are shown in Supplementary Table 3. At 2-year post-transplant overall survival was $62 \%$, LFS 54\%, and GRFS $37 \%$ while NRM
Table 1 Patient and transplant characteristics.

\begin{tabular}{|c|c|c|c|}
\hline & MRD negative & MRD positive & $\begin{array}{l}\text { Test } \\
p \text {-value }\end{array}$ \\
\hline Number & 749 & 293 & \\
\hline $\begin{array}{l}\text { Follow-up (surviving } \\
\text { patients): median } \\
\text { (range) (IQR) }\end{array}$ & $\begin{array}{l}24.6(0.5-132.5) \\
(7.7-54.3)\end{array}$ & $\begin{array}{l}26.9(0.8-121.5) \\
(8.2-60.4)\end{array}$ & 0.58 \\
\hline $\begin{array}{l}\text { Age at transplant: } \\
\text { median (range) (IQR) }\end{array}$ & $\begin{array}{l}49.4(18-78.1) \\
(37.7-58.8)\end{array}$ & $\begin{array}{l}49.8(19.5-72.9) \\
(37.6-58.4)\end{array}$ & 0.89 \\
\hline $\begin{array}{l}\text { Year of transplant: } \\
\text { median (range) }\end{array}$ & $\begin{array}{l}2012 \\
(2006-2016)\end{array}$ & $\begin{array}{l}2012 \\
(2006-2016)\end{array}$ & 0.31 \\
\hline $\begin{array}{l}\text { Time from diagnosis to } \\
\text { transplant: median } \\
\text { (range) (IQR) } \mathrm{m}\end{array}$ & $\begin{array}{l}18.2(3-200) \\
(13.5-27.1)\end{array}$ & $\begin{array}{l}15.9(3-200) \\
(11.6-21.8)\end{array}$ & $<10^{-4}$ \\
\hline Male patients & 390 (52.07\%) & $168(57.34 \%)$ & 0.13 \\
\hline Female patients & $359(47.93 \%)$ & $125(42.66 \%)$ & \\
\hline Donor male & $445(60.22 \%)$ & 189 (64.73\%) & 0.18 \\
\hline Donor female & 294 (39.78\%) & 103 (35.27\%) & \\
\hline Missing & 10 & 1 & \\
\hline $\begin{array}{l}\text { Female recipients and } \\
\text { male recipients of } \\
\text { male donors }\end{array}$ & $598(80.27 \%)$ & $243(83.22 \%)$ & 0.28 \\
\hline $\begin{array}{l}\text { Male recipients of } \\
\text { female donors }\end{array}$ & 147 (19.73\%) & 49 (16.78\%) & \\
\hline Missing & 4 & 1 & \\
\hline KPS $<80$ & $25(3.49 \%)$ & $8(2.86 \%)$ & 0.62 \\
\hline $\mathrm{KPS}>=80$ & 691 (96.51\%) & 272 (97.14\%) & \\
\hline missing & 33 & 13 & \\
\hline KPS $<90$ & $148(21.08 \%)$ & $63(22.74 \%)$ & 0.57 \\
\hline $\mathrm{KPS}>=90$ & 554 (78.92\%) & $214(77.26 \%)$ & \\
\hline Missing & 47 & 16 & \\
\hline Patient CMV negative & $248(34.35 \%)$ & $95(32.76 \%)$ & 0.63 \\
\hline Patient CMV positive & $474(65.65 \%)$ & $195(67.24 \%)$ & \\
\hline Missing & 27 & 3 & \\
\hline Donor CMV negative & 331 (45.84\%) & $146(51.59 \%)$ & 0.10 \\
\hline Donor CMV positive & 391 (54.16\%) & $137(48.41 \%)$ & \\
\hline Missing & 27 & 10 & \\
\hline $\begin{array}{l}\text { CMV donor negative/ } \\
\text { recipient negative }\end{array}$ & 177 (24.96\%) & $71(25.18 \%)$ & 0.24 \\
\hline $\begin{array}{l}\text { CMV donor positive/ } \\
\text { recipient negative }\end{array}$ & $66(9.31 \%)$ & $23(8.16 \%)$ & \\
\hline $\begin{array}{l}\text { CMV donor negative/ } \\
\text { recipient positive }\end{array}$ & $146(20.59 \%)$ & 74 (26.24\%) & \\
\hline
\end{tabular}


Table 1 continued

\begin{tabular}{|c|c|c|c|}
\hline & MRD negative & MRD positive & $\begin{array}{l}\text { Test } \\
p \text {-value }\end{array}$ \\
\hline $\begin{array}{l}\text { CMV donor positive/ } \\
\text { recipient positive }\end{array}$ & 320 (45.13\%) & $114(40.43 \%)$ & \\
\hline Missing & 40 & 11 & \\
\hline \multicolumn{4}{|l|}{ Cytogenetics } \\
\hline Good & 193 (48.61\%) & $102(53.4 \%)$ & 0.18 \\
\hline Intermediate & 188 (47.36\%) & 77 (40.31\%) & \\
\hline Adverse & $16(4.03 \%)$ & $12(6.28 \%)$ & \\
\hline Missing & 352 & 102 & \\
\hline FLT3 negative & 94 (63.09\%) & 66 (70.21\%) & 0.25 \\
\hline FLT3 positive & 55 (36.91\%) & 28 (29.79\%) & \\
\hline Missing & 600 & 199 & \\
\hline NPM1 negative & 42 (33.6\%) & $31(42.47 \%)$ & 0.21 \\
\hline NPM1 positive & 83 (66.4\%) & 42 (57.53\%) & \\
\hline Missing & 624 & 220 & \\
\hline MSD & $376(50.2 \%)$ & $134(45.73 \%)$ & 0.20 \\
\hline VUD HLA 10/10 & $373(49.8 \%)$ & 159 (54.27\%) & \\
\hline MAC & 430 (57.41\%) & 180 (61.43\%) & 0.24 \\
\hline $\mathrm{RIC}$ & 319 (42.59\%) & 113 (38.57\%) & \\
\hline
\end{tabular}

CMV cytomegalovirus, IQR interquartile range, KPS Karnofsky performance status, MAC myeloablative conditioning, MSD matched sibling donor, RIC reducedintensity conditioning, MUD matched unrelated donor.

was $18 \%$ and RI $29 \%$. At 100 days post-HCT, aGVHD grade III-IV was reported in $9 \%$ while at 2 years postHCT the cumulative incidence of cGVHD was $41 \%$, extensive in $20 \%$.

The median number of days to engraftment was shorter in MRD negative than MRD positive patients (15 vs. 16 days $P=0.001)$ but in both groups engraftment rates exceeded $98 \%(P=0.4)$ (Table 2$)$. Death in MRD positive vs. MRD negative patients was predominantly due to relapse ( $56 \%$ vs. $46 \%)$, GVHD ( $16 \%$ vs. $21 \%$ ), or infection (14\% vs. $21 \%)$ (Table 2 ).

In univariate analysis of outcomes at 2-year (Supplementary Table 4), MRD positive status at transplant was associated with excess relapse $(40 \%$ vs. $24 \% P<0.001)$, reduced LFS ( $46 \%$ vs. $57 \% P=0.001$ ), and worse GRFS (28\% vs. $41 \% P<0.001$ ) but overall equivalent survival at $62 \%$ compared to MRD negative patients. NRM and cGVHD rates were greater in MRD NEG than MRD POS (19\% vs. $14 \%$ and $42 \%$ vs. $39 \%$ ) but these did not reach statistical significance.

Survival and LFS were improved in patients whose characteristics included age less than the median of 49year, good risk cytogenetics at diagnosis, and longer times
Table 2 Transplant engraftment and toxicity in MRD negative vs. MRD positive patients.

\begin{tabular}{|c|c|c|c|}
\hline & $\begin{array}{l}\text { MRD } \\
\text { negative }\end{array}$ & $\begin{array}{l}\text { MRD } \\
\text { positive }\end{array}$ & $\begin{array}{l}\text { Test } \\
p \text {-value }\end{array}$ \\
\hline Engraftment failure & $8(1.07 \%)$ & $5(1.72 \%)$ & 0.40 \\
\hline Engrafted & 737 (98.93\%) & 286 (98.28\%) & \\
\hline Missing & 4 & 2 & \\
\hline $\begin{array}{l}\text { Time to neutrophils }>0.5 \times 10^{9} / \\
\mathrm{L} \text { median (range) (IQR) d }\end{array}$ & $\begin{array}{l}15(1-41) \\
(13-18)\end{array}$ & $\begin{array}{l}16(8-56) \\
(13-19)\end{array}$ & 0.00 \\
\hline Missing & 15 & 5 & \\
\hline \multicolumn{4}{|l|}{ Acute GVHD } \\
\hline Grade I & $113(15.48 \%)$ & $55(18.9 \%)$ & 0.13 \\
\hline Grade ॥ & $115(15.75 \%)$ & $42(14.43 \%)$ & \\
\hline Grade III & $33(4.52 \%)$ & $18(6.19 \%)$ & \\
\hline Grade IV & 22 (3.01\%) & $13(4.47 \%)$ & \\
\hline Grade unknown & $15(2.05 \%)$ & $1(0.34 \%)$ & \\
\hline Absence & $432(59.18 \%)$ & 162 (55.67\%) & \\
\hline Missing & 19 & 2 & \\
\hline No aGVHD II-IV & $545(76.22 \%)$ & $217(74.83 \%)$ & 0.64 \\
\hline aGVHD II-IV & $170(23.78 \%)$ & $73(25.17 \%)$ & \\
\hline Missing & 34 & 3 & \\
\hline No cGVHD & $430(63.52 \%)$ & $184(66.43 \%)$ & 0.39 \\
\hline cGVHD & 247 (36.48\%) & $93(33.57 \%)$ & \\
\hline Missing & 72 & 16 & \\
\hline Limited & $113(49.78 \%)$ & $29(32.58 \%)$ & \\
\hline Extensive & $114(50.22 \%)$ & $60(67.42 \%)$ & \\
\hline Missing & 20 & 4 & \\
\hline \multicolumn{4}{|l|}{ Causes of death } \\
\hline N & 281 & 120 & \\
\hline Cardiac toxicity & $3(1.16 \%)$ & $0(0 \%)$ & \\
\hline Haemorhage & $3(1.16 \%)$ & $1(0.88 \%)$ & \\
\hline sos & $4(1.54 \%)$ & $1(0.88 \%)$ & \\
\hline Infection & $55(21.24 \%)$ & $16(14.16 \%)$ & \\
\hline $\mathbb{I P}$ & $2(0.77 \%)$ & $4(3.54 \%)$ & \\
\hline GVHD & $54(20.85 \%)$ & $18(15.93 \%)$ & \\
\hline Original disease & $118(45.56 \%)$ & $63(55.75 \%)$ & \\
\hline Second malignancy & $4(1.54 \%)$ & $1(0.88 \%)$ & \\
\hline Other transplant related & $16(6.18 \%)$ & $9(7.96 \%)$ & \\
\hline Missing & 22 & 7 & \\
\hline
\end{tabular}

GVHD graft vs. host disease, MRD measurable residual disease, SOS sinusoidal obstructive syndrome.

from diagnosis to transplant (Supplementary Table 4). Relapse was also influenced by cytogenetic risk category and time to transplant but additional adverse factors were female donors for male recipients. NRM was only significantly affected by increasing patient age. $\mathrm{T}$ cell depletion had beneficial effects on day 100 rates of Grade III-IV GVHD, as well as 2-year rates of GRFS, cGVHD, and extensive cGVHD.

\section{Cox regression multivariate analysis}

Detailed outcomes of multivariate analysis are listed in Table 3. MRD Negative status conferred a significantly reduced risk of relapse (HR $0.67 \mathrm{CI} 0.44-0.73 P<0.001$ ) and extensive cGVHD (HR 0.57 CI 0.4-0.81 $P=0.0020$ 
Table 3 Cox regression analysis of transplant outcomes using variables derived from the outcome of univariate analysis or established risk factors.

\begin{tabular}{|c|c|c|c|c|c|c|c|c|c|c|c|c|}
\hline & \multicolumn{5}{|c|}{ Relapse } & \multicolumn{3}{|c|}{ NRM } & \multicolumn{3}{|c|}{ LFS } & \multirow[b]{2}{*}{$p$} \\
\hline & HR & $\mathrm{Cl}$ & & \multicolumn{2}{|c|}{$p$} & HR & $\mathrm{Cl}$ & $p$ & \multicolumn{2}{|r|}{ HR } & $\mathrm{Cl}$ & \\
\hline MRD negative at transplant & 0.57 & $0.44-0.73$ & & $1 e-c$ & & 1.31 & $0.91-0.90$ & 0.15 & & 0.76 & $0.62-0.94$ & 0.01 \\
\hline Age (per 10 years) & 0.96 & $0.86-1.07$ & & 0.42 & & 1.33 & $1.15-1.53$ & 0.00 & & 1.09 & $0.99-1.19$ & 0.07 \\
\hline RIC vs. MAC & 1.03 & $0.79-1.37$ & & 0.82 & & 0.82 & $0.58-1.17$ & 0.29 & & 0.93 & $0.75-1.16$ & 0.53 \\
\hline Good risk cytogenetics vs. all others & 0.62 & $0.46-0.83$ & & 0.00 & & 1.19 & $0.83-1.70$ & 0.36 & & 0.79 & $0.62-0.99$ & 0.04 \\
\hline Time from diagnosis to transplant & 0.98 & $0.97-0.99$ & & $<10^{-5}$ & & 1.00 & $0.99-1.00$ & 0.34 & & 0.99 & $0.98-0.99$ & $2 e-05$ \\
\hline VUD vs. MSD & 0.79 & $0.60-1.04$ & & 0.10 & & 1.11 & $0.77-1.60$ & 0.58 & & 0.90 & $0.72-1.13$ & 0.39 \\
\hline Female donor to male & 0.75 & $0.52-1.07$ & & 0.11 & & 1.30 & $0.88-1.89$ & 0.20 & & 0.94 & $0.72-1.22$ & 0.63 \\
\hline In vivo $\mathrm{TCD}$ & 1.35 & $1.02-1.79$ & & 0.03 & & 0.89 & $0.62-1.27$ & 0.51 & & 1.13 & $0.91-1.41$ & 0.27 \\
\hline Patient CMV positive & 1.03 & $0.78-1.36$ & & 0.82 & & 1.00 & $0.71-1.43$ & 0.98 & & 1.01 & $0.81-1.26$ & 0.9175 \\
\hline Donor CMV positive & 0.87 & $0.66-1.15$ & & 0.32 & & 1.39 & $0.98-1.97$ & 0.06 & & 1.06 & $0.85-1.31$ & 0.61 \\
\hline \multirow[t]{3}{*}{ Transplant center } & & & & 0.29 & & & & 0.16 & & & & 0.21 \\
\hline & \multicolumn{2}{|l|}{ OS } & & \multicolumn{4}{|c|}{ GRFS } & & \multicolumn{3}{|c|}{ AGVHD II-IV } & \\
\hline & HR & $\mathrm{Cl}$ & $p$ & & HR & $c$ & & $p$ & HR & & $\mathrm{Cl}$ & $p$ \\
\hline MRD negative at transplant & 1.00 & $0.77-1.20$ & 0.70 & & 0.69 & & -0.83 & $6 e-05$ & 0.91 & & $0.69-1.22$ & 0.52 \\
\hline Age (per 10 years) & 1.10 & $1.00-1.20$ & 0.058 & & 1.05 & & -1.13 & 0.25 & 0.94 & & $0.84-1.06$ & 0.29 \\
\hline RIC vs. MAC & 1.00 & $0.78-1.22$ & 0.83 & & 0.98 & & -1.18 & 0.80 & 0.84 & & $0.62-1.14$ & 0.26 \\
\hline Good risk cytogenetics vs. all others & 0.72 & $0.56-0.92$ & 0.01 & & 0.87 & & -1.06 & 0.16 & 1.04 & & $0.76-1.42$ & 0.80 \\
\hline Time from diagnosis to transplant & 0.99 & $0.980-0.99$ & 0.0 & & 1.00 & & -1.00 & 0.26 & 1.00 & & $1.00-1.01$ & 0.16 \\
\hline VUD vs. MSD & 0.95 & $0.75-1.20$ & 0.66 & & 1.14 & & -1.40 & 0.20 & 2.03 & & $1.46-2.81$ & $2 e-05$ \\
\hline Female donor to male & 1.06 & $0.76-1.32$ & 0.98 & & 1.08 & & -1.34 & 0.55 & 1.48 & & $1.06-2.07$ & 0.02 \\
\hline In vivo TCD & 1.10 & $0.88-1.38$ & 0.40 & & 0.61 & & -0.74 & $<10^{-5}$ & 0.62 & & $0.45-0.84$ & 0.00 \\
\hline Patient CMV positive & 0.95 & $0.76-1.20$ & 0.68 & & 1.17 & & -1.42 & 0.11 & 0.99 & & $0.73-1.33$ & 0.94 \\
\hline Donor CMV positive & 1.03 & $0.82-1.30$ & 0.78 & & 1.60 & & -1.28 & 0.56 & 1.17 & & $0.87-1.56$ & 0.30 \\
\hline \multirow[t]{3}{*}{ Transplant center } & & & 0.30 & & & & & 0.34 & & & & 0.15 \\
\hline & \multicolumn{3}{|c|}{ AGVHD III-IV } & & & \multicolumn{2}{|c|}{ CGVHD } & & \multicolumn{3}{|c|}{ EXT CGVHD } & \\
\hline & HR & $\mathrm{Cl}$ & & $p$ & & HR & $\mathrm{Cl}$ & $p$ & HR & & $\mathrm{Cl}$ & $p$ \\
\hline MRD negative at transplant & 0.65 & $0.41-1.03$ & & 0.06 & & 0.87 & $0.67-1.12$ & 0.26 & 0.5 & & $0.40-0.81$ & 0.00 \\
\hline Age (per 10 years) & 1.00 & $0.83-1.21$ & & 0.99 & & 1.02 & $0.92-1.14$ & 0.64 & 1.00 & & $0.87-1.16$ & 0.99 \\
\hline RIC vs. MAC & 1.22 & $0.74-2.01$ & & 0.44 & & 1.04 & $0.79-1.35$ & 0.80 & 0.92 & & $0.65-1.37$ & 0.76 \\
\hline Good risk cytogenetics vs. all others & 0.96 & $0.57-1.62$ & & 0.89 & & 1.09 & $0.77-1.31$ & 0.95 & 1.02 & & $0.71-1.47$ & 0.91 \\
\hline Time from diagnosis to transplant & 1.00 & $0.99-1.01$ & & 0.51 & & 1.00 & $0.10-1.09$ & 0.27 & 1.06 & & $0.10-1.01$ & 0.15 \\
\hline VUD vs. MSD & 1.65 & $0.98-2.77$ & & 0.06 & & 1.15 & $0.88-1.50$ & 0.32 & 1.7 & & $1.16-2.53$ & 0.01 \\
\hline Female donor to male & 1.12 & $0.63-2.10$ & & 0.70 & & 1.25 & $0.92-1.70$ & 0.16 & 1.45 & & $0.95-2.22$ & 0.08 \\
\hline In vivo $\mathrm{TCD}$ & 0.46 & $0.28-0.77$ & & 0.00 & & 0.43 & $0.33-0.56$ & $<10^{-5}$ & $0.1 \varepsilon$ & & $0.12-0.27$ & $<10^{-5}$ \\
\hline Patient CMV positive & 1.06 & $0.630-1.7$ & & 0.83 & & 0.96 & $0.73-1.24$ & 0.72 & 1.4 & & $0.96-2.09$ & 0.08 \\
\hline Donor CMV positive & 1.52 & $0.92-2.52$ & & 0.10 & & 1.27 & $0.97-1.64$ & 0.08 & 1.12 & & $0.78-1.67$ & 0.50 \\
\hline Transplant center & & & & 0.36 & & & & 0.10 & & & & 0.12 \\
\hline
\end{tabular}

cGVHD chronic graft vs. host disease, $a G V H D$ acute graft vs. host disease, CMV cytomegalovirus, GRFS graft vs. host disease and relapse-free survival, GVHD graft vs. host disease, LFS leukemia-free survival, MAC myeloablative conditioning, MRD measurable residual disease, MSD matched sibling donor, NRM non-relapse mortality, OS overall survival, RIC reduced-intensity conditioning, TCD T cell depletion, MUD volunteer unrelated donor.

which resulted in improved LFS (HR 0.76 CI $0.62-0.94$ $P=0.01$ ) and GRFS (HR 0.69 CI $0.57-0.83 P<0.001$ ) but no change in OS. Other established factors had predictable effects on outcomes. Thus, OS and LFS were both enhanced in patients with good risk cytogenetics or prolonged time interval from diagnosis to transplant. While TCD was associated with improved GRFS, there was no concomitant improvement in OS or LFS, probably due to the increased risk of relapse. The use of unrelated donors or female donors for male recipients increased the risk of aGVHD grades II-IV and extensive cGVHD but TCD reduced the risks of all forms of aGVHD and cGVHD. NRM increased with advancing age.

\section{Analysis of MRD POS vs. MRD NEG in MSD or MUD}

Finally, a subgroup analysis was performed according to the donor type, MSD or MUD, in univariate 
(Supplementary Table 5) and then Cox regression analysis (Supplementary Tables 6, 7).

When an MSD was used, the significant benefits of MRD negative status were maintained for relapse (HR 0.45 CI $0.31-0.65 P<0.001$ ), LFS (HR 0.65 CI 0.48-0.89 $P=0.006$ ), GRFS (HR $0.58 \mathrm{CI} 0.44-0.76 P<0.001$ ) and extensive cGVHD (HR 0.43 CI $0.26-0.71 P<0.001$ ) but OS was similar to patients with MRD POS status (Supplementary Table 6). As expected, NRM increased with age and was reduced by the use of RIC HCT, while TCD improved GRFS and cGVHD rates, but otherwise patient age, conditioning intensity, cytogenetic status at diagnosis, and TCD made no significant impact on transplant outcomes. Time to transplant from original diagnosis maintained its predictive effect on OS, LFS, NRM, and relapse (Supplementary Table 6). Interestingly, CMV seropositive patients and donors were associated with excess rates of extensive CGVHD and aGVHD grades III-IV, respectively.

The use of an HLA 10/10 MUD appeared to compensate for the presence of MRD since transplant outcomes were similar in MRD POS vs. MRD NEG recipients (Supplementary Table 7). Increasing age was linked to increased NRM while good risk cytogenetics predicted superior OS, LFS, and relapse rates. Relapse rates and LFS were beneficially related to long periods from diagnosis to transplant. Conditioning intensity had no apparent effect on transplant outcomes. The use of TCD reduced all forms of GVHD and improved GRFS but at the expense of OS, probably reflecting trends towards increased relapse and worse LFS. Patients and donors who were CMV positive were associated with excess cGVHD and NRM, respectively.

\section{Discussion}

We report the first large series of transplant outcomes in 1042 adults with AML in CR2 with an established MRD status at transplant. These patients were transplanted in multiple centers, mostly European, who reported details of transplants to the EBMT. Of note, the MSD and MUD groups were balanced except for cytogenetic risk (more good risk in the MSD group, conditioning (more TBI in MSD), and as expected for GVHD prevention.

The Seattle group showed that MRD status prior to HCT was predictive of outcome in 70 patients with AML $\mathrm{CR}^{31}$. These patients had a median age of 42.3 (range 2.1-72.6) years and all were treated with MAC HCT plus, in some cases, in vivo $\mathrm{T}$ cell depletion with antithymocyte globulin. The EBMT cohort differs from North American subjects by excluding children and by including RIC, as well as MAC HCTs, and collecting GRFS status. MRD status was assessed by multiparameter flow cytometry immunophenotyping and/or by PCR-based assays according to center preference ${ }^{47}$.
Unfortunately, we did not have individual information on the method used that would allow us to compare the outcome between PCR and flow cytometry. Despite this limitation, we found that MRD negative patients had a substantially reduced risk of relapse at $24.1 \%$ compared to $39.8 \%$ in MRD positive recipients, and this translated into a superior LFS and GRFS (Table 2 and Supplementary Table 4). However, MRD status had no independent impact on OS although MRD negative status was associated with a trend to increased NRM which may have partially offset the improved LFS (Table 3 and Supplementary Table 4). The largest effects on OS were exerted by the established risk factors of cytogenetic status at diagnosis and the time interval from diagnosis to transplant and these also impacted LFS (Table 3 and Supplementary Table 4). The relatively few patients with adverse risk cytogenetics reflect standard practice to offer transplant in CR1.

The fitness of patients for transplant may be evaluated by a combination of performance status (PS), comorbidity index, and frailty assessment ${ }^{56-58}$. Due to a lack of data we were only able to study the impact of Karnofsky (PS) and found no impact on transplant outcomes in multivariate analysis (Supplementary Table 4). This may possibly reflect a more tailored transplant approach by centers to patients with lower PS.

We also looked at the effect of TCD as this is used extensively in Europe. In this cohort, TCD was associated with increased relapse rates and improved GRFS but no difference in LFS and OS. Increased RI in association with TCD in older patients with AML CR1 MRD NEG status at transplant has also been reported ${ }^{35}$. However, the use of ATG as TCD has not generally been associated with significant increases in relapse risk in other studies performed by the EBMT Acute Leukemia Working Party, although these have been predominantly performed in patients with AML CR1 rather than CR $2^{47,59,60}$. The use of TCD in vivo with anti-thymocyte globulin or Alemtuzumab requires further prospective study due to conflicting results in published studies which vary in disease stage, absolute lymphocyte count at the time of TCD, dose schedule, and associated conditioning regimen ${ }^{47,61-65}$.

We have previously studied the effect of conditioning intensity in AML CR1 and found that MAC HCT was superior to RIC HCT only in patients $<50$ years who were MRD pos at $\mathrm{HCT}^{35}$. Overall, in this cohort, we found no benefit to increased intensity of conditioning. Patients under the median age of 49.4 years had better NRM, LFS, and OS at the expense of higher aGVHD rates than older counterparts in univariate analysis (Supplementary Table 4). In multivariate analysis, however, the only effect of increasing age was to increase NRM. This is in keeping with our previous study of conditioning intensity in HCT AML CR2 where there is 
no impact on survival in patients under 50 years but an increase in NRM for patients of 50 or more years, particularly following MAC $\mathrm{HCT}^{45}$.

The adverse impact of MRD POS status on RI and LFS in the whole group was also seen when patients in receipt of sibling donors were studied but interestingly this effect was not evident in those patients receiving MUD HCT. This sub-group analysis supports the possibility that the use of a volunteer donor confers an enhanced graft vs leukemia effect and may be preferable to a sibling donor in AML CR2 MRD POS, thus contributing to the ongoing debate about the relative merits of sibling vs. volunteer donors $^{66-68}$. The advent of high-resolution HLA typing has reduced NRM in MUD HCT and may allow the graft vs. leukemia effect to be studied in a more homogenous setting in future studies ${ }^{69}$.

While we did not see any impact of MRD status on OS in this study, in contrast to the effects of cytogenetic risk group and time from diagnosis to transplant, we did not have details of post-HCT salvage regimens such as targeted therapy with FLT3 inhibitors or donor lymphocyte infusions (DLI). We also lacked data on the precise methodology and validation criteria of the MRD assays used by each center. Since this was a registry analysis we cannot exclude bias on the part of the centers with respect to the decision to transplant. In addition, it was not possible to ascertain the precise duration of CR1 or the FLT3 and NPM1 status at diagnosis for all patients.

Monitoring of MRD status peri-HCT is rapidly becoming standard clinical practice for patients with AML with consequent recourse to DLI and investigational drugs were available for patients with evidence of MRD. However, international standardization of MRD assays will be key to future insights into the implications of MRD.

\section{Acknowledgements}

We thank all European Group for Blood and Marrow Transplantation (EBMT) centers and national registries for contributing patients to the study and data managers for their superb work. The study was supported by the European Blood and Marrow Transplantation funded by annual subscription from the constituent transplant centers.

\footnotetext{
Author details

'Leeds Teaching Hospitals Trust, St James's University Hospital, Leeds LS9 7TF, United Kingdom. ${ }^{2}$ Hematology and Bone Marrow Transplantation Division, Chaim Sheba Medical Center, Tel-Hashomer, Sackler School of Medicine, TelAviv University, 6997801 Tel-Aviv, Israel. ${ }^{3}$ EBMT Paris study office/CEREST-TC, Paris, France. ${ }^{4}$ University Hospital Bristol NHS Foundation Trust, London, United Kingdom. ${ }^{5}$ Department of Bone Marrow Transplantation, West German Cancer Center, University Hospital of Essen, Essen, Germany. ${ }^{6}$ Hematologie/ Transplantation, Hôpital St Louis, Paris, CEDEX 10, France. ${ }^{7}$ Kapadokya BMT Center, Kayseri, Turkey. ${ }^{8}$ Department of Hematology, Hemostasis, Oncology and Stem Cell Transplantation, Hannover Medical School, Hannover, Germany. ${ }^{9}$ Hematology Service, Institute of Hematology and Blood Transfusion, Prague, Czech Republic. ${ }^{10}$ Department of Haematology, Rigshospitalet, Copenhagen, Denmark. "'CHU de Lille, LIRIC, INSER U995, Université de Lille, Lille, France.

${ }^{12}$ Manchester Royal Infirmary, Manchester, United Kingdom. ${ }^{13}$ Acute Leukemia
}

Working Party, European Society for Blood and Marrow Transplantation Paris Study Office/European Center for Biostatistical and Epidemiological Evaluation in Hematopoietic Cell Therapy (CEREST-TC), Paris, France. ${ }^{14}$ Hôpital Saint Antoine, INSERM UMR 938, Paris, France; Université Pierre et Marie Curie, Paris, France. ${ }^{15}$ Hematology Division, Chaim Sheba Medical Center, Tel Hashomer, Israel

\section{Conflict of interest}

The authors declare no competing interests.

\section{Publisher's note}

Springer Nature remains neutral with regard to jurisdictional claims in published maps and institutional affiliations.

Supplementary information The online version contains supplementary material available at https://doi.org/10.1038/s41408-021-00479-3.

Received: 31 May 2020 Revised: 7 November 2020 Accepted: 1 December 2020

Published online: 12 May 2021

\section{References}

1. Cornelissen, J. J. et al. Results of a HOVON/SAKK donor versus no-donor analysis of myeloablative HLA-identical sibling stem cell transplantation in first remission acute myeloid leukemia in young and middle-aged adults: benefits for whom? Blood 109, 3658-3666 (2007).

2. Gooley, T. A. et al. Reduced mortality after allogeneic hematopoietic-cell transplantation. N. Engl. J. Med. 363, 2091-2101 (2010).

3. Döhner, H. et al. Diagnosis and management of AML in adults: 2017 ELN recommendations from an international expert panel. Blood 129, 424-447 (2016).

4. Cornelissen, J. J. et al. The European LeukemiaNet AML Working Party consensus statement on allogeneic HSCT for patients with AML in remission: an integrated-risk adapted approach. Nat. Rev. Clin. Oncol. 9, 579-590 (2012).

5. Del Principe, M. I. et al. Minimal residual disease in acute myeloid leukemia of adults: determination, prognostic impact and clinical applications. Mediterr. J. Hematol. Infect. Dis. 8, e2016052 (2016).

6. Buckley, S. A. et al. Minimal residual disease prior to allogeneic hematopoietic cell transplantation in acute myeloid leukemia: a meta-analysis. Haematologica 102, 865-873 (2017).

7. Mosna, F., Capelli, D. \& Gottardi, M. Minimal residual disease in acute myeloid leukemia: still a work in progress? J. Clin. Med. 6, 57 (2017).

8. Hourigan, C. S. \& Karp, J. E. Minimal residual disease in acute myeloid leukaemia. Nat. Rev. Clin. Oncol. 10, 460 (2013).

9. Voso, M. T. et al. MRD in AML: the role of new techniques. Front. Oncol. 9, 655 (2019).

10. Sievers, E. L. et al. Immunophenotypic evidence of leukemia after induction therapy predicts relapse: results from a prospective Children's Cancer Group study of 252 patients with acute myeloid leukemia. Blood 101, 3398-3406 (2003).

11. Inaba, H. et al. Comparative analysis of different approaches to measure treatment response in acute myeloid leukemia. J. Clin. Oncol. 30, 3625-3632 (2012).

12. Buccisano, F. et al. Cytogenetic and molecular diagnostic characterization combined to postconsolidation minimal residual disease assessment by flow cytometry improves risk stratification in adult acute myeloid leukemia. Blood 116, 2295-2303 (2010).

13. Reading, C. L. et al. Expression of unusual immunophenotype combinations in acute myelogenous leukemia. Blood 81, 3083-3090 (1993).

14. Terwijn, M. et al. High prognostic impact of flow cytometric minimal residual disease detection in acute myeloid leukemia: data from the HOVON/SAKK AML 42A study. J. Clin. Oncol. 31, 3889-3897 (2013).

15. Grimwade, D. \& Freeman, S. D. Defining minimal residual disease in acute myeloid leukemia: which platforms are ready for "prime time"? Blood 124, 3345-3355 (2014).

16. Czyz, A. \& Nagler, A. The role of measurable residual disease (MRD) in hematopoietic stem cell transplantation for hematological malignancies focusing on acute leukemia. Int. J. Mol. Sci. 20, 5362 (2019). 
17. San Miguel, J. F. et al. Early immunophenotypical evaluation of minimal residual disease in acute myeloid leukemia identifies different patient risk groups and may contribute to postinduction treatment stratification. Blood $\mathbf{9 8}$ 1746-1751 (2001).

18. Feller, N. et al. MRD parameters using immunophenotypic detection methods are highly reliable in predicting survival in acute myeloid leukaemia. Leukemia 18, 1380-1390 (2004).

19. Kern, W. et al. Determination of relapse risk based on assessment of minimal residual disease during complete remission by multiparameter flow cytometry in unselected patients with acute myeloid leukemia. Blood 104, 3078-3085 (2004).

20. MRD-AML-BFM Study Group, Langebrake C. et al. Residual disease monitoring in childhood acute myeloid leukemia by multiparameter flow cytometry: the MRD-AML-BFM Study Group. J. Clin. Oncol. 24, 3686-3692 (2006).

21. Kern, W., Haferlach, C., Haferlach, T. \& Schnittger, S. Monitoring of minimal residual disease in acute myeloid leukemia. Cancer.112, 4-16 (2008).

22. Maurillo, L. et al. Toward optimization of postremission therapy for residual disease-positive patients with acute myeloid leukemia. J. Clin. Oncol. 26, 4944-4951 (2008)

23. van der Velden, V. H. J. et al. Clinical significance of flowcytometric minimal residual disease detection in pediatric acute myeloid leukemia patients treated according to the DCOG ANLL97/MRC AML12 protocol. Leukemia 24 1599-1606 (2010).

24. Paietta, E. Minimal residual disease in acute myeloid leukemia: coming of age. Hematol. Am. Soc. 2012, 35-42 (2012).

25. Loken, M. R. et al. Residual disease detected by multidimensional flow cytometry signifies high relapse risk in patients with de novo acute myeloid leukemia: a report from Children's Oncology Group. Blood 120, 1581-1588 (2012).

26. Freeman, S. D. et al. Prognostic relevance of treatment response measured by flow cytometric residual disease detection in older patients with acute myeloid leukemia. J. Clin. Oncol. 31, 4123-4131 (2013).

27. Buckley, S. A., Appelbaum, F. R. \& Walter, R. B. Prognostic and therapeutic implications of minimal residual disease at the time of transplantation in acute leukemia. Bone Marrow Transplant. 48, 630-641 (2013).

28. Walter, R. B. et al. Impact of pretransplantation minimal residual disease, as detected by multiparametric flow cytometry, on outcome of myeloablative hematopoietic cell transplantation for acute myeloid leukemia. J. Clin. Oncol. 29, 1190-1197 (2011).

29. Leung, W. et al. High success rate of hematopoietic cell transplantation regardless of donor source in children with very high-risk leukemia. Blood $\mathbf{1 1 8}$ 223-230 (2011).

30. Leung, W. et al. Detectable minimal residual disease before hematopoietic cell transplantation is prognostic but does not preclude cure for children with very-high-risk leukemia. Blood 120, 468-472 (2012).

31. Walter, R. B. et al. Significance of minimal residual disease before myeloablative allogeneic hematopoietic cell transplantation for AML in first and second complete remission. Blood 122, 1813-1821 (2013).

32. Oran, B. et al. Pre-transplantation minimal residual disease with cytogenetic and molecular diagnostic features improves risk stratification in acute myeloid leukemia. Haematologica 102, 110-117 (2017).

33. Walter, R. B. et al. Comparison of minimal residual disease as outcome predictor for AML patients in first complete remission undergoing myeloablative or nonmyeloablative allogeneic hematopoietic cell transplantation. Leukemia 29, 137-144 (2015).

34. Nomdedéu J. F. et al. Bone marrow WT1 levels in allogeneic hematopoietic stem cell transplant $(\mathrm{HCT})$ for acute myeloid leukemia and myelodysplasia: clinically relevant time-points and 100 copies threshold value. Biol. Blood Marrow Transplant. 24, 55-63 (2017).

35. Gilleece, M. H. et al. Measurable residual disease, conditioning regimen intensity, and age predict outcome of allogeneic hematopoietic cell transplantation for acute myeloid leukemia in first remission: A registry analysis of 2292 patients by the Acute Leukemia Working Party European Society of Blood and Marrow Transplantation. Am. J. Hematol. 93, 1142-1152 (2018).

36. Forman, S. J. \& Rowe, J. M. The myth of the second remission of acute leukemia in the adult. Blood. 121, 1077-1082 (2013).

37. Gale, R. P. \& Lazarus, H. M. Correcting 2 more myths regarding transplants for AML in second remission. Blood. 123, 794 (2014).

38. Gale, R. P., Wiernik, P. H. \& Lazarus, H. M. Should persons with acute myeloid leukemia have a transplant in first remission? Leukemia 28, 1949-1952 (2014).
39. Ganzel, C. et al. Very poor long-term survival in past and more recent studies for relapsed AML patients: the ECOG-ACRIN experience. Am. J. Hematol. 93 1074-1081 (2018)

40. Breems, D. A. et al. Prognostic index for adult patients with acute myeloid leukemia in first relapse. J. Clin. Oncol. 23, 1969-1978 (2005).

41. Burnett, A. K. et al. Curability of patients with acute myeloid leukemia who did not undergo transplantation in first remission. J. Clin. Oncol. 31, 1293-1301 (2013).

42. Kurosawa, S. et al. Prognostic factors and outcomes of adult patients with acute myeloid leukemia after first relapse. Haematologica 95, 1857-1864 (2010).

43. Hospital, M.-A. et al. Core-binding factor acute myeloid leukemia in first relapse: a retrospective study from the French AML Intergroup. Blood 124, 1312-1319 (2014).

44. Weisdorf, D. J. et al. Allogeneic transplantation for advanced acute myeloid leukemia: the value of complete remission. Cancer.123, 2025-2034 (2017).

45. Gilleece, M. H. et al. Allogeneic haemopoietic transplantation for acute myeloid leukaemia in second complete remission: a registry report by the Acute Leukaemia Working Party of the EBMT. Leukemia 34, 87-99 (2020).

46. European Society for Blood and Marrow Transplantation [Internet]. [cited 2018 Mar 30]. www.ebmt.org.

47. Nagler, A. et al. The impact of anti-thymocyte globulin on the outcomes of Patients with AML with or without measurable residual disease at the time of allogeneic hematopoietic cell transplantation. Leukemia 34, 1144-1153 (2019).

48. Grimwade, D. et al. Refinement of cytogenetic classification in acute myeloid leukemia: determination of prognostic significance of rare recurring chromosomal abnormalities among 5876 younger adult patients treated in the United Kingdom Medical Research Council trials. Blood 116, 354-365 (2010).

49. Bacigalupo, A. et al. Defining the intensity of conditioning regimens: working definitions. Biol. Blood Marrow Transplant. 15, 1628-1633 (2009).

50. Shimoni, A. et al. Intravenous busulfan compared with treosulfan-based conditioning for allogeneic stem cell transplantation in acute myeloid leukemia: a study on Behalf of the Acute Leukemia Working Party of European Society for Blood and Marrow Transplantation. Biol. Blood Marrow Transplant. 24, 751-757 (2018).

51. Saraceni, F. et al. Thiotepa-busulfan-fludarabine compared to busulfanfludarabine for sibling and unrelated donor transplant in acute myeloid leukemia in first remission. Oncotarget. 9, 3379-3393 (2018).

52. Shulman, H. M. et al. Chronic graft-versus-host syndrome in man. A long-term clinicopathologic study of 20 Seattle patients. Am. J. Med. 69, 204-217 (1980).

53. Ruggeri, A., Labopin, M., Ciceri, F., Mohty, M. \& Nagler, A. Definition of GVHDfree, relapse-free survival for registry-based studies: an ALWP-EBMT analysis on patients with AML in remission. Bone Marrow Transplant. 51, 610-611 (2016).

54. Hougaard, P. Frailty models for survival data. Lifetime Data Anal. 1, 255-273 (1995).

55. Andersen, P. K., Klein, J. P. \& Zhang, M. J. Testing for centre effects in multicentre survival studies: a Monte Carlo comparison of fixed and random effects tests. Stat. Med. 18, 1489-1500 (1999).

56. Sorror, M. et al. Hematopoietic cell transplantation-comorbidity index and Karnofsky performance status are independent predictors of morbidity and mortality after allogeneic nonmyeloablative hematopoietic cell transplantation. Cancer 112, 1992-2001 (2008).

57. Cohen, H. J. et al. Frailty as determined by a comprehensive geriatric assessment-derived deficit-accumulation index in older patients with cancer who receive chemotherapy. Cancer 122, 3865-3872 (2016).

58. Holmes, H. M. et al. Optimal screening for geriatric assessment in older allogeneic hematopoietic cell transplantation candidates. J. Geriatr. Oncol. 5 422-430 (2014).

59. Baron, F. et al. Anti-thymocyte globulin as graft-versus-host disease prevention in the setting of allogeneic peripheral blood stem cell transplantation: a review from the Acute Leukemia Working Party of the European Society for Blood and Marrow Transplantation. Haematologica 102, 224-234 (2017).

60. Ofran, Y. et al. Anti-thymocyte globulin for graft-versus-host disease prophylaxis in patients with intermediate- or high-risk acute myeloid leukaemia undergoing reduced-intensity conditioning allogeneic stem cell transplantation in first complete remission-a survey on behalf of the Acute Leukaemia Working Party of the European Society for Blood and Marrow Transplantation. Br. J. Haematol. 184, 643-646 (2019).

61. Walker, I. et al. Pretreatment with anti-thymocyte globulin versus no antithymocyte globulin in patients with haematological malignancies undergoing haemopoietic cell transplantation from unrelated donors: a randomised, controlled, open-label, phase 3, multicentre trial. Lancet Oncol. 17, 164-173 (2016). 
62. Finke, J. et al. Long-term outcomes after standard graft-versus-host disease prophylaxis with or without anti-human-T-lymphocyte immunoglobulin in haemopoietic cell transplantation from matched unrelated donors: final results of a randomised controlled trial. Lancet Haematol. 4, e293-e301 (2017).

63. Soiffer, R. J. et al. Prospective, randomized, double-blind, phase III clinical trial of anti-T-lymphocyte globulin to assess impact on chronic graft-versus-host disease-free survival in patients undergoing HLA-matched unrelated myeloablative hematopoietic cell transplantation. J. Clin. Oncol. 35, 4003-4011 (2017).

64. Kröger, N. et al. Antilymphocyte globulin for prevention of chronic graftversus-host disease. N. Engl. J. Med. 374, 43-53 (2016).

65. Chakraverty, R. et al. Impact of in vivo alemtuzumab dose before reduced intensity conditioning and HLA-identical sibling stem cell transplantation: pharmacokinetics, GVHD, and immune reconstitution. Blood 116, 3080-3088 (2010).

66. Ringdén, $\mathrm{O}$. et al. The graft-versus-leukemia effect using matched unrelated donors is not superior to HLA-identical siblings for hematopoietic stem cell transplantation. Blood 113, 3110-3118 (2009).

67. Ruggeri, A. et al. Unrelated donor versus matched sibling donor in adults with acute myeloid leukemia in first relapse: an ALWP-EBMT study. J. Hematol. Oncol. 9, 89 (2016).

68. Weisdorf, D. Which donor or graft source should you choose for the strongest GVL? Is there really any difference. Best Pract. Res. Clin. Haematol. 26, 293-296 (2013).

69. Lee, S. J. et al. High-resolution donor-recipient HLA matching contributes to the success of unrelated donor marrow transplantation. Blood 110, 4576-4583 (2007). 